\title{
Productivity, Energy Prices and the Great Moderation: A New Link*
}

\author{
Rajeev Dhawan \\ Georgia State University, rdhawan@gsu.edu \\ Karsten Jeske \\ Federal Reserve Bank of Atlanta, karsten.jeske@atl.frb.org \\ Pedro Silos \\ Federal Reserve Bank of Atlanta, pedro.silos@atl.frb.org
}

February 15, 2008

\begin{abstract}
We study how total factor productivity (TFP), energy prices and the great moderation are linked. First, we estimate a joint stochastic process for the energy price and TFP and establish that until 1982:II, energy prices negatively affected productivity. This spill-over has since disappeared. Second, we show that within the framework of a Dynamic Stochastic General Equilibrium (DSGE) model, the disappearance of this energy-productivity spillover generates the significantly lower volatility of output and its components. Specifically, the change in the joint stochastic process accounts for close to 70 percent of the moderation in output volatility.
\end{abstract}

JEL codes: C22, E32, Q43

Keywords: Bayesian Estimation, Productivity, Energy Price, Great Moderation, Business Cycles

\footnotetext{
*Very preliminary, we appreciate comments.
} 


\section{Introduction}

Our research is motivated by the fact that output volatility in the United States has declined significantly since the mid 1980s, as first documented by Kim and Nelson (1999) and McConnell and Perez-Quiros (2000). Stock and Watson (2002) also document the fall in volatility in other important macro variables such as consumption, investment, and hours worked. This drop in volatilities of output and most other macro variables is an artifact economists also call the "Great Moderation." ${ }^{1}$ In this paper we study the link between energy prices and total factor productivity (TFP) and the role it plays in accounting for the great moderation. ${ }^{2}$

Why would the fluctuations in energy prices be important for the great moderation? Looking at the energy price and its relationship with business cycles reveals that the deep recessions in 1973-74 and 1980-82 were preceded by large energy price spikes. ${ }^{3}$ However, the energy price drop in 1986 did not spark a significant acceleration in GDP growth and, likewise, the sustained rise in energy prices since 2002 has not yet led to a recession. ${ }^{4}$ Our hypothesis from these observations is that a link between energy prices and business cycles existed in the early period, say, before 1982, but has since disappeared, potentially accounting for the lower volatility of macro variables. This motivates the empirical analysis where we estimate a joint stochastic process for quarterly energy prices and TFP using Bayesian estimation methods. We explicitly model a spill-over effect from the energy price innovations to TFP and the magnitude of this spill-over varies over time. Specifically, we allow for a breakpoint from one regime into another, and the timing of this break itself is a parameter to be estimated. We find the second quarter of 1982 (1982:II) to be the estimated breakpoint. Before 1982:II, innovations in the process for the energy price had a significant and negative spill-over into TFP. This spill-over disappeared afterwards.

Next we use a dynamic stochastic general equilibrium (DSGE) modeling framework to evaluate the impact of the changing nature of the joint stochastic process for energy prices and TFP on key macro volatilities. Specifically, we take the Kim and Loungani (1992) model, which incorporates energy use as a complement to fixed capital on the production side, and simulate it with the pre and post 1982:II specification for the joint stochastic process for the price of energy and TFP. We show that the absence of the spill-over effect after 1982:II reduces output volatility by about 34 percent. $^{5}$ Given that the actual drop in output volatility after 1982 was about 55 percent, the changing nature of the stochastic process accounts for about 61 percent of the great

\footnotetext{
${ }^{1}$ Then-Federal Reserve Governor Ben Bernanke coined this phrase at the Eastern Economic Association Meetings in 2004 .

${ }^{2}$ See Owyang, Piger and Wall (2007) for a survey of competing explanations in the great moderation debate.

${ }^{3}$ See Hamilton $(1983,2003)$ and Hamilton and Herrera (2004) for evidence on the link between energy prices and business cycles.

${ }^{4}$ The residential construction activity has dropped sharply from its peak in 2006 but the overall economy is not in a recession according to the NBER definition at the time of writing this paper.

${ }^{5}$ Consumption and investment volatilities also declined by a similar magnitude.
} 
moderation in output volatility.

One can object that the significant drop in the share of energy use in GDP since the early 80's can directly account for the reduced volatility, without the added link of energy prices on productivity. Thus, we simulate the model without an energy-productivity spill-over but with different energy shares calibrated to the observed energy to output ratios in the pre and post 1982:II time-periods. This experiment generates a drop in output volatility of only 5 percent, compared to the 55 percent observed in the data. Thus, a drop in the energy share accounts for a marginal proportion (less than 10 percent) of the great moderation. Of course, if we simulate the model not only with different stochastic processes but also with different energy shares calibrated to the early and late period, we enhance the drop in output volatility to 37 percent, bringing the share of the great moderation accounted for by the model to 68 percent.

Our paper proceeds as follows. Section 2 details the specification and estimation of the joint stochastic process for TFP and energy. Section 3 introduces the DSGE model and the calibration of the remaining parameters. Section 4 presents the numerical results in the benchmark case with fixed energy shares and also for the model with varying energy shares. Finally, section 5 concludes.

\section{Econometric Setup}

We model the energy price time series as an $\operatorname{ARMA}(1,1)$ process, which is the typical structure in business cycle models with energy use ${ }^{6}$

$$
p_{t}=\rho^{p} p_{t-1}+\epsilon_{t}^{p}+\xi \epsilon_{t-1}^{p}
$$

The $\epsilon_{t}^{p}$ is a zero-mean innovation to the energy price shock assumed to be normally distributed with a variance $\sigma_{p}^{2}$.

We deviate from the usual $\mathrm{AR}(1)$ specification for the productivity process, as the innovation in our study is to assume spill-over effects from energy prices into TFP. As a result, we specify a process in which productivity $z$ depends on past values of innovations to the energy prices,

$$
z_{t}=\rho^{z} z_{t-1}+\epsilon_{t}^{z}+\sum_{\tau=1}^{4} \gamma_{t}^{\tau} \epsilon_{t-\tau}^{p}
$$

The innovations to the productivity shock $\epsilon_{t}^{z}$ will also be distributed Normal with a variance $\sigma_{z}^{2}$ and the degree (and direction) of the spill-over will be given by the values of $\gamma_{t}^{\tau}$. Note the subscript $t$ in the spill-over parameter $\gamma$ : we assume that the degree of spill-over effects from

\footnotetext{
${ }^{6}$ See, for example, Kim and Loungani (1992) and Dhawan and Jeske (2006).
} 
energy prices to productivity has changed in the last 50 years. Specifically, we model this as a one-time change with an unknown date $t^{*}$, which we will treat as another parameter to be estimated. As a consequence, the spill-over parameters will take on values $\gamma_{1}=\left(\gamma_{1}^{1}, \gamma_{1}^{2}, \gamma_{1}^{3}, \gamma_{1}^{4}\right)$ in the first part of the sample and $\gamma_{2}=\left(\gamma_{2}^{1}, \gamma_{2}^{2}, \gamma_{2}^{3}, \gamma_{2}^{4}\right)$ in the second part. This means the productivity process has the following form

$$
z_{t}=\rho^{z} z_{t-1}+\epsilon_{t}^{z}+\sum_{\tau=1}^{4} \gamma_{t}^{\tau} \epsilon_{t-\tau}^{p}, \quad \epsilon_{t}^{z} \sim N\left(0, \sigma_{z}^{2}\right)
$$

where

$$
\gamma_{t}=\left(\gamma_{t}^{1}, \gamma_{t}^{2}, \gamma_{t}^{3}, \gamma_{t}^{4}\right)= \begin{cases}\gamma_{1} & \text { if } t \leq t^{\star} \\ \gamma_{2} & \text { if } t>t^{\star}\end{cases}
$$

We use data for quarterly energy price and productivity $\left\{p_{t}, z_{t}\right\}_{t=1}^{T}$ to estimate the parameters of the two stochastic processes, where $T$ is the sample size. Data cover the period from 1970 to 2005. Appendix A has the details on how we construct the quarterly series for TFP and the energy price.

We model the one-time change in $\gamma$ as the transition of a two-state Markov process into an absorbing state. Assume that the value of $\gamma$ is driven by a latent variable $S_{t}, S_{t} \in\{0,1\}$ for any $t$, which follows a Markov chain with transition probability:

$$
\Pi_{\epsilon}=\left[\begin{array}{cc}
q & 1-q \\
0 & 1
\end{array}\right]
$$

We let the data inform us whether there has been a transition into a state in which $S_{t}=1$. If a transition occurs, we denote the date at which occurs as $t^{*}$. The goal of the procedure is to estimate the vector of parameters and latent variables: $\left\{\rho^{p}, \xi, \sigma_{p}^{2}, \rho^{z}, \gamma_{1}, \gamma_{2}, \sigma_{z}^{2},\left\{S_{t}\right\}_{t=1}^{T}, q, t^{*}\right\}$.

The procedure can be split into two steps: the estimation of the energy price process and the the estimation of the productivity process.

In the energy price process there is a total of three parameters to estimate. Denote the vector of the three parameters $\theta_{p}=\left\{\rho^{p}, \xi, \sigma_{p}\right\}$ and $f_{p}\left(\gamma_{p}\right)$ the prior distribution over these parameters. To construct the likelihood function we first cast the $\operatorname{ARMA}(1,1)$ as a state-space system:

$$
\begin{gathered}
\zeta_{t+1}=\left[\begin{array}{rr}
\rho^{p} & 0 \\
1 & 0
\end{array}\right] \zeta_{t}+w_{t+1} \\
p_{t}=\left[\begin{array}{ll}
1 & \xi
\end{array}\right] \zeta_{t}+v_{t+1}
\end{gathered}
$$

The likelihood, $L\left(\left\{p_{t}\right\}_{t=1}^{T} \mid \theta_{p}\right)$ is then constructed as described in Hamilton (1994), (Ch. 13, p. 
$385)$ which makes use of the Kalman filter to integrate out the latent vector $\zeta_{t}$. Once we compute the likelihood, we find the posterior distribution, $p\left(\theta_{p} \mid\left\{p_{t}\right\}_{t=1}^{T}\right)$, by coupling the likelihood and the prior: $f_{p}\left(\theta_{p} \mid\left\{p_{t}\right\}_{t=1}^{T}\right) \propto L\left(\left\{p_{t}\right\}_{t=1}^{T} \mid \theta_{p}\right) f_{p}\left(\theta_{p}\right)$. We obtain draws from this posterior using wellknown sampling methods. Specifically, we use a Metropolis-Hastings algorithm (see e.g. Robert and Casella (1999)).

Next we estimate the productivity process using the a time series of $\left\{\epsilon_{t}^{p}\right\}_{t=1}^{T}$ as data. Denote by $\theta_{z}$ the vector of parameters $\left\{\rho_{z}, \gamma_{1}, \gamma_{2}, \sigma_{z}, q\right\}$. First, we endow $\theta_{z}$ with a prior distribution $f_{z}\left(\theta_{z}\right)$. To compute the likelihood for the TFP process, there is the obvious difficulty that the vector $\left\{S_{t}\right\}_{t=1}^{T}$ is not observed. If it were observed, the likelihood function would be:

$$
L\left(\left\{z_{t}\right\}_{t=1}^{T} \mid \theta_{z},\left\{S_{t}\right\}_{t=1}^{T}\right)=\prod_{t=5}^{T} \frac{1}{\sqrt{2 \pi \sigma_{z}^{2}}} \exp \left\{-\frac{1}{2 \sigma_{z}^{2}} e_{t}^{2}\right\}
$$

with $e_{t}=z_{t}-\rho_{z} z_{t-1}-\sum_{\tau=1}^{4} \gamma_{1}^{\tau} \epsilon_{t-\tau}^{p}$ for $S_{t}=0$ and $e_{t}=z_{t}-\rho_{z} z_{t-1}-\sum_{\tau=1}^{4} \gamma_{2}^{\tau} \epsilon_{t-\tau}^{p}$ for $S_{t}=1$. Given that we do not know $\left\{S_{t}\right\}_{t=1}^{T}$, we use a filtering (and smoothing) procedure similar to that described in Kim and Nelson (1999, Chapters 4 and 9). The algorithm consists of the following steps:

1. Denoting by $g\left(S_{t} \mid z_{t}, z_{t-1}, \theta_{z}\right)$ the mass function for $S_{t}$ (i.e. the filtered probabilities), compute the likelihood function using Hamilton's (1989) filter. This gives $\left\{g\left(S_{t} \mid z_{t}, z_{t-1}, \theta_{z}\right)\right\}_{t=5}^{T}$.

2. Couple this likelihood with the prior for $\theta_{z}$ to obtain a draw from the posterior $f_{z}\left(\theta_{z} \mid\left\{z_{t}\right\}_{t=1}^{T}\right)$.

3. For $t=T-1, T-2 \ldots, 5$, compute the smoothed probabilities given by:

$$
g\left(S_{t} \mid\left\{z_{t}\right\}, S_{t+1}\right) \propto g\left(S_{t+1} \mid S_{t}\right) g\left(S_{t} \mid\left\{z_{t}\right\}\right)
$$

4. Repeating the above three steps $M$ times, we obtain $M^{7}$ draws from the posterior distribution for $\theta_{z}$ and $\left\{S_{t}\right\}$.

We report the prior distribution for the parameters in Table 1 . We have used (truncated ${ }^{8}$ Normal distributions for $\rho^{p}, \rho^{z}, \xi, \gamma_{1}$, and $\gamma_{2}$; Gamma distributions for $\sigma_{p}^{2}$ and $\sigma_{z}^{2}$; and a Beta distribution for $q$. These distributions are fairly uninformative except for the sign restriction in the $\gamma$ 's to be able to identify the two regimes. The prior distribution for $q$ implies a mean

\footnotetext{
${ }^{7}$ We drew $M=30000$ draws for the estimation of both the energy price and the TFP process. We discarded the first 5000 .

${ }^{8}$ We have used with indicator variables to determine the region of truncation. For example $\chi_{\left|\rho^{p}\right|<1}$ takes the value of zero whenever the absolute value of $\rho_{p}$ is greater than one.
} 
Table 1: Prior Distributions

\begin{tabular}{cc}
\hline \hline Parameter & Distribution \\
\hline$\rho^{p}$ & $N\left(0.9,1.5^{2}\right) \chi_{\left|\rho^{p}\right|<1}$ \\
$\xi$ & $N\left(0,0.4^{2}\right)$ \\
$\sigma_{p}^{2}$ & Gamma $(0.0001,1)$ \\
$\rho^{z}$ & $N\left(0.9,1.5^{2}\right) \chi_{\left|\rho^{z}\right|<1}$ \\
$\left\{\gamma_{1}^{i}\right\}_{i=1}^{4}$ & $N\left(0,0.15^{2}\right) \chi_{\gamma_{1}^{i}>0}$ \\
$\left\{\gamma_{2}^{i}\right\}_{i=1}^{4}$ & $N\left(0,0.15^{2}\right) \chi_{\gamma_{2}^{i}<0}$ \\
$\sigma_{z}^{2}$ & $\operatorname{Gamma}(0.0001,1)$ \\
$q$ & $\operatorname{Beta}(0.6,0.4)$ \\
\hline \hline
\end{tabular}

of 0.50 and a standard deviation of 0.48 and the prior distribution for the variances of the shocks has a low mean $\left(9.35 \times 10^{-5}\right)$ but a large standard deviation $(0.009)$. Table 2 shows our estimation results. We date the time of the change at the second quarter of 1982, but this being an estimate, there is some uncertainty around it as well. A $90 \%$ posterior region is bounded by the third quarter of 1979 and the second quarter of 1985. In the first subperiod the spill-over parameters $\gamma$ are significantly less than zero. For the second subperiod, however, zero is well within two posterior standard deviations of the mean, so we can conclude that the spill-over effect is only significant during the first period (i.e. the period for which $t<t^{*}$ ), in which higher energy prices due to positive innovations affect TFP negatively. As is expected the parameters driving persistence in energy prices and TFP are large, while the variance of the innovations is small for TFP and large for energy prices.

Table 2: Estimation Results

\begin{tabular}{ccc}
\hline \hline Parameter & Posterior Mean & Post. Std. Dev. \\
\hline$\rho^{p}$ & 0.960 & 0.034 \\
$\xi$ & 0.451 & 0.102 \\
$\sigma_{p}^{2}$ & 0.001 & $1.59 \times 10^{-4}$ \\
$\rho^{z}$ & 0.915 & 0.034 \\
$\gamma_{1}$ & {$[-0.091,-0.020,-0.060,-0.051]$} & {$[0.032,0.016,0.033,0.031]$} \\
$\gamma_{2}$ & {$[0.016,0.024,0.029,0.016]$} & {$[0.011,0.013,0.015,0.011]$} \\
$\sigma_{z}^{2}$ & $3.25 \times 10^{-5}$ & $3.94 \times 10^{-6}$ \\
$q$ & 0.978 & 0.019 \\
$t^{*}$ & $1982: \mathrm{II}$ & $\mathrm{N} / \mathrm{A}$ \\
\hline \hline
\end{tabular}




\section{DSGE Model Setup}

In the previous section we showed that there is a statistically significant difference between the parameters of the TFP process in the two subperiods. How significant are the two different shock processes for TFP in an economic sense? To answer this question we feed the stochastic process for the energy price and the two alternative specifications for the productivity process into a Dynamic Stochastic General Equilibrium (DSGE) model. The model is identical to the one described in Kim and Loungani (1992). Households have preferences over consumption $c$ and leisure equal to the normalized total hours less hours worked $h$,

$$
U=E_{0} \sum_{t=0}^{\infty} \beta^{t}\left[\varphi \log c_{t}+(1-\varphi) \log \left(1-h_{t}\right)\right]
$$

Output $y$ is produced by a representative firm that combines hours, capital stock $k$ and energy $e$. Production is also subject to a stochastic total productivity shock $z$,

$$
y_{t}=z_{t}\left(\eta k_{t-1}^{\nu}+(1-\eta) e_{t}^{\nu}\right)^{\alpha / \nu} h_{t}^{1-\alpha}
$$

The elasticity of substitution between capital and energy is $\frac{1}{1-\nu}$. Consequently, the production function displays complementarity between capital and energy when $\nu<0$. Energy has to be imported at the relative price $p_{t}$ and capital depreciates at a rate $\delta$, thus the economy's resource constraint is

$$
c_{t}+k_{t}-(1-\delta) k_{t-1}+p_{t} e_{t}=y_{t}
$$

and the capital stock evolves according to

$$
k_{t}=(1-\delta) k_{t}+i_{t}
$$

The social planner thus solves the following optimization problem

$$
\max E_{0} \sum_{t=0}^{\infty} \beta^{t}\left[\varphi \log c_{t}+(1-\varphi) \log \left(1-h_{t}\right)\right]
$$

subject to

$$
c_{t}+k_{t}-(1-\delta) k_{t-1}+p_{t} e_{t}=z_{t}\left(\eta k_{t-1}^{\nu}+(1-\eta) e_{t}^{\nu}\right)^{\alpha / \nu} h_{t}^{1-\alpha}
$$


and

$$
\begin{aligned}
& p_{t}=\rho^{p} p_{t-1}+\epsilon_{t}^{p}+\xi \epsilon_{t-1}^{p} \\
& z_{t}=\rho_{z} z_{t-1}+\epsilon_{t}^{z}+\sum_{\tau=1}^{4} \gamma^{\tau} \epsilon_{t-\tau}^{p}
\end{aligned}
$$

We nned to assign values for the following parameters: $\beta, \varphi, \alpha, \nu, \eta, \delta$. Throughout the paper, we set the parameters $\alpha, \beta$ and $\nu$ at 0.36, 0.99, and -0.70 as in Kim and Loungani (1992). We calibrate the remaining parameters to match the targets $k / y=12, e / y=0.0544$ and $h=0.3$. To this end, we derive the first order conditions in Appendix section B.1 and set parameters to ensure that in the model steady state generates the targets specified above. Appendix section B.2 provides the details of this calibration process. We report the parameters from this calibration exercise in Table 3.

\begin{tabular}{cl} 
Table 3: Model Paramete \\
\hline \hline Parameter & Value \\
\hline$\beta$ & 0.9900 \\
$\alpha$ & 0.3600 \\
$\nu$ & -0.7000 \\
$\varphi$ & 0.3376 \\
$\eta$ & 0.9959 \\
$\delta$ & 0.0154 \\
\hline \hline
\end{tabular}

\section{Results}

\subsection{Benchmark}

Table 4: Volatility in the data versus model

\begin{tabular}{lcccccc}
\hline \hline & \multicolumn{2}{c}{ Early period } & \multicolumn{2}{c}{ Late period } & \multicolumn{2}{c}{ Percentage drop } \\
& $(1970: I-1982: \mathrm{I})$ & \multicolumn{2}{c}{$(1982: I I-2006: I V)$} & \multicolumn{2}{c}{ in volatility } \\
\hline \multirow{3}{*}{ Output } & Data & Model & Data & Model & Data & Model \\
Consumption & 2.07 & 1.87 & 0.94 & 1.25 & -54.89 & -33.50 \\
Investment & 6.73 & 0.37 & 0.73 & 0.24 & -55.96 & -34.40 \\
Hours & 1.86 & 1.15 & 3.55 & 6.00 & -47.33 & -34.82 \\
\hline \hline
\end{tabular}

Note: Volatilities refer to the standard deviation of log-deviations from HP-filtered series

$$
(\lambda=1600) \text {. }
$$


We take first order necessary conditions in Appendix B.1 and compute a log-linear approximation around the deterministic steady state. We do so for the two alternative sets of $\gamma$ parameters in the stochastic process for TFP to simulate the economy under the two regimes.

In Table 4, we report the volatilities of output, consumption, investment and hours worked in the data and in the model in the two different periods (pre and post 1982:II). Volatility in the data dropped across the board, by about 55 percent for output and consumption, 47 percent for fixed investment and 36 percent for hours worked. In the model we generate a drop in output volatility of almost 34 percent. Thus, 61 percent of the moderation is explained by change in the spill-over effect of energy price into TFP. Consumption, investment and hours volatility also drops by about 33 to 35 percent. $^{9}$

\subsection{Different energy shares}

As Dhawan and Jeske (2007) point out, the energy share in the production has diminished in the last decades. We compute the energy shares in the two subperiods and recalibrate the model to account for the two alternative calibration targets ${ }^{10}$. This changes the values for both $\eta$ and $\delta$, as detailed in Table 5 .

Table 5: Different calibration targets for energy shares and corresponding parameter values

\begin{tabular}{lcc}
\hline \hline & $\begin{array}{c}\text { Early period } \\
(1970: \mathrm{I}-1982: \mathrm{I})\end{array}$ & $\begin{array}{c}\text { Late period } \\
(1982: \mathrm{II}-2006: \mathrm{IV})\end{array}$ \\
\hline$e / y$ & 0.0678 & 0.0436 \\
$\eta$ & 0.9938 & 0.9973 \\
$\delta$ & 0.0142 & 0.0163 \\
\hline \hline
\end{tabular}

We first simulate the economy without a spill-over $\left(\gamma_{1}=\gamma_{2}=[0,0,0,0]\right)$ but with different energy shares. Then we simulate the economy with the spill-over and different energy shares. The first experiment determines whether the change in the energy share alone can account for the great moderation. The second experiment determines by how much we enhance our results in the benchmark economy when, in addition to the spill-over, we also allow for a change in the energy share.

Changing only the shares but not the stochastic process between the two periods does not generate a large drop in volatilities of macro variables as documented in Table 6. Output volatility drops by less than 5 percent, consumption volatility by 3 percent, which is much less than what

\footnotetext{
${ }^{9}$ Notice that the consumption volatility in the model is much lower than in the data. As we know from Cooley and Prescott (1995), DSGE models have a hard time generating enough consumption volatility.

${ }^{10}$ The exercise of changing the energy share and computing the volatilities for the two regimes was also performed by Nakov and Pescatori (2007).
} 
is observed in the data. The investment volatility drops considerably more, though still not close to the drop observed in the data. The reason why the investment volatility drops much more than consumption is because of the complementarity of capital and energy in production.

As expected, the model with the spill-over effect and different energy shares explains an even larger decline in the volatility than in the benchmark with fixed energy shares as we demonstrate in Table 7. Output volatility drops by about 37 percent, which accounts for 68 percent of the observed drop in the data, slightly higher than the 61 percent drop in the benchmark calibration. It appears that the reduction in the energy share helps explain some of the great moderation but compared to the spill-over mechanism its impact is of secondary importance.

Table 6: Volatility in the data versus model: Different energy shares, no spill-over

\begin{tabular}{lcccccc}
\hline \hline & \multicolumn{2}{c}{ Early period } & \multicolumn{2}{c}{ Late period } & \multicolumn{2}{c}{$\begin{array}{c}\text { Percentage drop } \\
\text { in volatility }\end{array}$} \\
& $(1970: I-1982: \mathrm{I})$ & \multicolumn{2}{c}{$(1982: I I-2006: I V)$} & \multicolumn{2}{c}{ Data } & Model \\
Output & 2.07 & 1.28 & 0.94 & 1.22 & -54.89 & -4.89 \\
Consumption & 1.67 & 0.25 & 0.73 & 0.25 & -55.96 & -3.27 \\
Investment & 6.73 & 6.73 & 3.55 & 5.54 & -47.33 & -17.62 \\
Hours & 1.86 & 0.78 & 1.20 & 0.73 & -35.59 & -5.44 \\
\hline \hline
\end{tabular}

Note: Volatilities refer to the standard deviation of log-deviations from HP-filtered series $(\lambda=1600)$.

Table 7: Volatility in the data versus model: Different energy shares and spill-over from the energy price to productivity

\begin{tabular}{lcccccc}
\hline \hline & \multicolumn{2}{c}{$\begin{array}{c}\text { Early period } \\
(1970: I-1982: I)\end{array}$} & \multicolumn{2}{c}{ Late period } & \multicolumn{2}{c}{$\begin{array}{c}\text { Percentage drop } \\
\text { in volatility }\end{array}$} \\
\hline \multirow{3}{*}{ Output } & Data & Model & Data & Model & Data & Model \\
Consumption & 2.07 & 1.95 & 0.94 & 1.22 & -54.89 & -37.48 \\
Investment & 6.73 & 0.39 & 0.73 & 0.24 & -55.96 & -37.77 \\
Hours & 1.86 & 1.20 & 1.20 & 0.74 & -35.59 & -38.14 \\
\hline \hline
\end{tabular}

Note: Volatilities refer to the standard deviation of log-deviations from HP-filtered series $(\lambda=1600)$.

\section{Concluding Remarksand Discussion}

When simulating DSGE models, researchers normally assume that the shocks hitting the economy are orthogonal. In our paper we show that innovations to energy prices and total factor 
productivity (TFP) have not been orthogonal before 1982:II. In contrast, the two stochastic processes have been close to independent since then. We demonstrate that the change in the structure of the stochastic processes can account for more than 61 percent of the drop in output volatility. Adding the reduced share of energy use to this framework increases the explanatory power of the model to 68 percent.

There are two opposing views in the economics literature on the importance of energy shocks. The empirical literature, for example in Hamilton (1983, 2003) and Hamilton and Herrera (2004), finds a significant link between oil price shocks and business cycles. On the other hand, the DSGE literature, as in Kim and Loungani (1992) and Dhawan and Jeske (2006), shows that total factor productivity (TFP) is the main source of business cycle fluctuations, while energy prices play almost no role. Our paper reconciles these findings from the econometric and DSGE literature in the following sense. The recessions in the 1970s and 1980s occurred not because of the direct effect of the energy price hikes but because of their spill-over effect on productivity as our model simulation results demonstrate. After 1982:II, this spill-over effect disappears which then reduces the volatility of TFP and thus that of macro variables. Thus, our results support Arias, Hansen and Ohanian (2007) who demonstrate that the drop in TFP volatility within the framework of a DSGE model is the main reason for the drop in output volatility.

This raises the question: what caused the moderation of TFP volatility? Arias, Hansen and Ohanian are silent on what caused the decline in TFP volatility, though they show that a previously conjectured channel, unmeasured factor utilization in the construction of the TFP series, is likely not the cause. Furthermore they rule out government spending shocks and preference shocks as the source of output volatility moderation. ${ }^{11}$ Hence, one can view our paper as providing a new stylized fact in the great moderation debate in the sense that we demonstrate that the drop in TFP volatility has to do with the reduced spill-over from energy price shocks.

Currently, we do not take a position on either the source of the spill-over in the early period or the reason for its sudden disappearance in the 1980s. Rather, our aim is to establish this new stylized fact and encourage researchers to theoretically account for our empirical findings. For future research it will interesting to determine possible causes for the energy to TFP spill-over in the early period as well as reasons for the sudden disappearance after 1982. One possible route is to model the price controls during the Carter and Nixon years that were abolished in the early 1980s. Price controls and the resulting rationing prevent the factor energy from being used in the most productive way. Without explicitly modeling this friction the rationing would show up as lower TFP in response to an energy price shock.

\footnotetext{
${ }^{11}$ Leduc and Sill (2007) show that although a change in the monetary policy decision rule (Taylor Rule) can indeed account for lower inflation volatility, the drop in output volatility comes chiefly from the drop in TFP volatility.
} 


\section{References}

[1] Arias, Andres, Gary D. Hansen and Lee E. Ohanian. 2007. Why have business cycles fluctuations become less volatile? Economic Theory. 32 (July): 43-58.

[2] Collard, F. and M. Juillard. 2001. "Accuracy of stochastic perturbation methods: The case of asset pricing models." Journal of Economic Dynamics and Control 25 (June-July): 97999 .

[3] Cooley, Thomas F. and Edward C. Prescott. 1995. "Economic Growth and Business Cycles" In Frontiers of Business Cycle Research, Princeton, NJ.

[4] Dhawan, Rajeev and Karsten Jeske. 2007. "What Determines the Output Drop after an Energy Price Increase: Household or Firm Energy Share?" Federal Reserve Bank of Atlanta Working Paper 2007-20.

[5] Dhawan, Rajeev and Karsten Jeske. Forthcoming. "Energy Price Shocks and the Macroeconomy: The Role of Consumer Durables" Journal of Money, Credit, and Banking.

[6] Hamilton, James. 1994. "Time Series Analysis." Princeton University Press. Princeton, New Jersey.

[7] Hamilton, James and Anna M. Herrera. 2004. "The Oil Shocks and Aggregate Macroeconomic Behavior: The Role of Monetary Policy." Journal of Money, Credit, and Banking 36 (April): 265-86.

[8] Kim, In-Moo and Prakash Loungani. 1992. "The role of energy in real business cycle models" Journal of Monetary Economics 29: 173-89.

[9] Kim, C.-J. and C.R. Nelson (1999), "Has the U.S. Economy Become More Stable? A Bayesian Approach Based on a Markov-Switching Model of the Business Cyclemodels." The Review of Economics and Statistics 81, 608 - 616.

[10] Leduc, Sylain and Keith Sill. 2007. "Monetary policy, oil shocks, and TFP: Accounting for the decline in US volatility." Review of Economic Dynamics 10, No. 5 (October). pp. 595-614.

[11] McConnell, M.M and G. Perez-Quiros. 2000. "Output Fluctuations in the United States: What has Changed Since the Early 1980's" American Economic Review, Vol. 90, No. 5, 1464-1476.

[12] Nakov, A. and A. Pescatori. 2007. "Oil and the Great Moderation" Banco de España Working Paper 2007-0.735.

[13] Owyang, Michael T., Jeremy Piger and Howard J. Wall. 2007. "A State-Level Analysis of the Great Moderation" Federal Reserve Bank of St. Louis Working Paper.

[14] Stock, James H. and Mark W. Watson, "Has the Business Cycle Changed and Why?" NBER Macroeconomics Annual, 2002, Vol. 17 Issue 1, p. 159-218.

\section{Appendix}




\section{A Data}

We construct the data series as following. The real energy price is the natural logarithm of the quarterly price index of gasoline, fuel energy, natural gas and electricity from the BEA, adjusted by the GDP deflator.

To construct the TFP we use quarterly output data from the BEA and the hours series from the Establishment survey. Notice that

$$
z_{t}=y_{t}\left(\eta k_{t-1}^{\nu}+(1-\eta) e_{t}^{\nu}\right)^{-\alpha / \nu} h_{t}^{-1+\alpha}
$$

As in Cooley and Prescott (1995) we assume that capital is fixed $\left(k_{t-1}=\bar{k}\right)$ at the quarterly frequency when computing TFP. Firm energy use exists only at the annual frequency, so as a first approximation we assume that $e_{t}$ is fixed as well. Then, just as in Cooley and Prescott (1995), we construct TFP as

$$
\tilde{z}_{t}=\tilde{y}_{t}-(1-\alpha) \tilde{h}_{t}
$$

where the tilde stand for log-deviations from the trend. Next, we also computed TFP by explicitly taking into account firm energy use by a) converting the annual energy use into quarterly data by interpolation and b) by using the quarterly household energy use as a proxy for firm energy use. Since the resulting TFP time series were very similar to the one constructed by assuming fixed energy use(correlation coefficient close to 0.98), and the estimation of the stochastic processes in section 2 were essentially identical, we kept the same procedure as in Cooley and Prescott for TFP calculation. Finally, as a sensitivity check we also generated artificial data from the model and compared the series for $\tilde{z}_{t}$ with that of the $\tilde{z}_{t}$ constructed via equation $\left(\mathrm{A}-1^{\prime}\right)$ and again found the two series to be very similar, with a correlation coefficient of 0.95 .

\section{B First order conditions and calibration}

\section{B.1 First order necessary optimality conditions:}

The following equations, together with the definition of the stochastic processes in equations (1) and (2), define the dynamics of the model:

1. Consumption vs. leisure

$$
(1-\varphi) \frac{1}{1-h_{t}}=\varphi \frac{1}{c_{t}} w_{t}
$$

2. Definition of the wage

$$
w_{t}=(1-\alpha) \frac{y_{t}}{h_{t}}
$$

3. Definition of the rate of return on capital

$$
r_{t}=y_{t} \alpha \frac{\eta k_{t-1}^{\nu-1}}{\eta k_{t-1}^{\nu}+(1-\eta) e_{t}^{\nu}}
$$

4. Energy price equals marginal product of energy

$$
p_{t}=y_{t} \alpha \frac{(1-\eta) e_{t}^{\nu-1}}{\eta k_{t-1}^{\nu}+(1-\eta) e_{t}^{\nu}}
$$


5. Euler Equation

$$
1=\beta E\left\{\frac{c_{t}}{c_{t+1}}\left(1+r_{t+1}-\delta\right)\right\}
$$

6. Definition of output

$$
y_{t}=z_{t}\left(\eta k_{t-1}^{\nu}+(1-\eta) e_{t}^{\nu}\right)^{\alpha / \nu} h_{t}^{1-\alpha}
$$

7. Evolution of capital

$$
i_{t}=k_{t}-(1-\delta) k_{t-1}
$$

8. Resource constraint

$$
c_{t}+i_{t}+p_{t} e_{t}=y_{t}
$$

\section{B.2 Calibration}

We have to pin down the following six parameters: $\beta, \varphi, \alpha, \nu, \eta, \delta$. We fix $\alpha, \beta$ and $\nu$ to the same values as in Kim and Loungani (1992) and Dhawan and Jeske (2006) and calibrate the remaining parameters $\varphi, \eta$ and $\delta$ to match our targets for $k / y, e / y$ and $h$. In steady state:

$$
\begin{aligned}
p & =y \alpha \frac{(1-\eta) e^{\nu-1}}{\eta k^{\nu}+(1-\eta) e^{\nu}} \\
& =\alpha \kappa_{k}^{-1} \frac{(1-\eta) e^{\nu-1}}{\eta k^{\nu-1}+(1-\eta) e^{\nu} k^{-1}} \\
& =\alpha \kappa_{k}^{-1} \frac{1-\eta}{\eta\left(\frac{\kappa_{k}}{\kappa_{e}}\right)^{\nu-1}+(1-\eta)\left(\frac{\kappa_{k}}{\kappa_{e}}\right)^{-1}} \\
& =\alpha \frac{1-\eta}{\eta \kappa_{e}^{1-\nu} \kappa_{k}^{\nu}+(1-\eta) \kappa_{e}}
\end{aligned}
$$

where $\kappa_{k}=k / y, \kappa_{e}=e / y$. Then

$$
p\left(\eta \kappa_{e}^{1-\nu} \kappa_{k}^{\nu}+(1-\eta) \kappa_{e}\right)=\alpha-\alpha \eta
$$

Rearranging

$$
\eta\left(p \kappa_{e}^{1-\nu} \kappa_{k}^{\nu}+\alpha-p \kappa_{e}\right)=\alpha-p \kappa_{e}
$$

Thus,

$$
\eta=\frac{\alpha-p \kappa_{e}}{p \kappa_{e}^{1-\nu} \kappa_{k}^{\nu}+\alpha-p \kappa_{e}}
$$

Next solve for $r$ :

$$
r=\frac{k}{y} \frac{k^{\nu-1}}{\eta+(1-\eta)\left(\frac{e}{k}\right)^{\nu}} \alpha \eta
$$

and from the Euler equation:

$$
\delta=r-\frac{1}{\beta}+1
$$


Finally, from the labor vs consumption equation, combined with the wage equation:

$$
\begin{aligned}
\frac{(1-\varphi)}{\varphi} & =\frac{1-h}{h}(1-\alpha) \frac{y}{c} \\
& =\frac{1-h}{h}(1-\alpha)\left(1-\delta \kappa_{k}-p \kappa_{e}\right)^{-1}
\end{aligned}
$$

Thus,

$$
\varphi=\left[1+\frac{1-h}{h}(1-\alpha)\left(1-\delta \kappa_{k}-p \kappa_{e}\right)^{-1}\right]^{-1}
$$

